\title{
IRREDUCIBLE CONTINUA AND GENERALIZATION OF HEREDITARILY UNICOHERENT GONTINUA BY MEANS OF MEMBRANES
}

\author{
R. F. DICKMAN, L. R. RUBIN and P. M. SWINGLE *
}

(Received 6 May 1964)

\section{Introduction}

In [2] we defined an irreducible $B(J)$-cartesian membrane, and used this to obtain a characterization of an $n$-sphere by generalizing the definition of simple closed curve given by Theorem 1.2 below. There $B(J)$ is a class $A(n)$ of $(n-1)$-spheres, but here it is a class of mutually homeomorphic continua. In Theorem 1.1 we give a definition of hereditarily unicoherent continua and generalize this in Section 3 by means of $B(J)$-cartesian membranes. To do this we paraphrase by a translation some of Wilder's work in [7]. In his Unified Topology [8: p. 674] he gives a principle: "The connectedness of a domain is a special case of the bounding properties of its $i$-cycles". We substitute the element $J$ of $B(J)$ for the $i$-cycle and for "bound" we substitute that " $J$ membrane-bases an irreducible $B(J)$-cartesian membrane. The very nature of an $i$-cycle seems to limit the complexity of the point set studied, although the restriction to "nice" manifolds is due partly to the difficulty of the subject matter treated. There are similar difficulties here, but also advantages, in the very general set-theoretic approach by means of $B(J)$-membranes.

\section{Characterization of topological spaces by the number of irreducible continua between points}

The Theorems here give set-theoretic characterizations of hereditarily unicoherent continua, simple closed curves, hereditarily indecomposable continua, arcs and dendrites. We mean by a continuum a closed and connected set as in [6]; we denote the null set by $\emptyset$. In this Section a continuum $M$ has hereditarily a property $P$ if every subcontinuum of $M$ has property $P$.

Theorem 1. Let $X$ be a compact Hausdorff continuum and let $a, b \in X$ such that $a \neq b$. Then:

* This work was done under National Science Foundation grant G 19672. 
(1.1) $X$ is hereditarily unicoherent if and only if, for each pair $a$ and $b$, there exists exactly one subcontinum of $X$ irreducible from $a$ to $b$;

(1.2) $X$ is a simple closed curve if and only if, for each pair $a$ and $b$, there exist exactly two distinct subcontinua $I_{1}$ and $I_{2}$ irreducible from $a$ to $b$ and $X=I_{1}+I_{2}$, and if $I^{\prime}$ is another subcontinum of $X$ irreducible from a to $b$, then either $I^{\prime}=I_{1}$ or $I^{\prime}=I_{2}$, and

(1.3) $X$ is hereditarily indecomposable if and only if, for each pair $a$ and $b$ and any proper subcontinum $I$ of $X$ irreducible from $a$ to $b$, if $c \in X-I$, and $M$ is a subcontinum of $X$ irreducible from $c$ to $b$, then $I \subset M$.

We proved (1.2) as Theorem 1 of [2]. The proof of (1.1) follows easily from the definitions, and parallels that of its generalization as Theorem 4 of Section 3: for its partial use in semigroups, see Hunter in [4: p. 363]. The proof of (1.3) follows easily from definitions and the use of Theorem 43, in [6: p. 15; see also p. 58].

We define the set-valued set-function $T=T(A, Y)$, for $A \subset Y$ and $Y \subset X$, as follows. Let $Q$ be an open subset of $Y$ and $W$ be a subcontinuum of $Y$. Then:

$y \notin T(A, Y)$ if and only if there exist $Q$ and $W$ such that $y \in Q \subset W \subset Y-A$.

For examples and fundamental properties see [1], where $T(A, X)=$ $T(A)$ for $X$ the imbedding space.

LEMMA 1. Let $X$ be a compact Hausdorff continuum irreducible from $a$ to $b$, let $x \in X-a-b, a \neq b$, and let $x(a)$ and $x(b)$ be subcontinua of $X$ irreducible from $x$ to $a$ and $x$ to $b$ respectively: then $a$ necessary and sufficient condition that $X$ be a simple continuous arc is that both $T(x, x(a))=x$ and $x=T(x, x(b))$ for every $x \in X$.

Proof. The necessity follows easily from the definitions. The sufficiency will follow from Corollary 16.1 of $[1:$ p. 274], if we prove $T(x, X)=x$ which we now do. Take $y \in x(a)-x$. Since $T(x, x(a))=x$, there exist in $x(a)$ an open set $Q$ and a continuum $W$ such that $y \in Q \subset W \subset x(a)-x$. Consider the case $y \notin x(b)$ : then take $Q^{\prime}=Q \cdot(X-x(b))$. Since $x(a)$ and $x(b)$ are continua with $a \in x(a)$ and $b \in x(b), x(a)+x(b)=X$. Thus $X-x(b) \subset x(a)$ and so $Q^{\prime}$ is an open subset of $X-x(b)$, which itself is open in $X$. Therefore $Q^{\prime}$ is open in $X$ and so $y \in Q^{\prime} \subset W \subset x(a)-x \subset X-x$; thus $y \notin T(x, X)$.

Consider now the case $y \in x(b)$; then $y \in x(a) \cdot x(b)$.

By Theorem 12 of $[1:$ p. 272] $T(x, x(a))=\operatorname{cl}(I(a, x(a)))$, and so by hypothesis $I(a, x(a))=x$. There exists a subcontinuum $C$ of $x(a)$ which contains $y$ and $a$ but not $x$ by definition [1: p. 272] of Kuratowski's $I(a, x(a))$. Similarly there exists a subcontinuum $C^{\prime}$ of $x(b)$ which contains $y$ and $b$ but not $x$. But then $C+C^{\prime}$ is a proper subcontinuum of $X$ containing 
$a$ and $b$ which contradicts the irreducibility of $X$. Thus $y \notin x(b)$, and so $T(x, X)=x$ for all $x \in X$ and hence $X$ is an arc.

THEOREM 2. If $X$ is a compact Hausdorff continuum such that for $n \geqq 2$ and for each $a, b \in X$ there are $n$ but not more than $n$ distinct subcontinua $X_{i}(i=1,2, \cdots, n)$ of $X$ irreducible from $a$ to $b$ and $X$ is the essential union of the $X_{i}$, then each $X_{i}$ is an arc.

Proof. We recall in the definition $[10:$ p. 88] of essential union that $X=X_{1}+X_{2}+\cdots+X_{n}$ and each $X_{i}$ contains an essential point which is not in the union of the remaining $X_{j}$. Note that an essential point in an $X_{i}$ has an essential neighborhood in $X_{i}$, since $\bigcup X_{j}(j \neq i)$ is closed. Now let $a, b \in X$ and $\left\{X_{1}\right\}(i=1,2, \cdots, n)$ be the $n$ irreducible continua of the hypothesis. For some $i \leqq n$, let $x \in X_{i}-a-b$. Let $x(a)$ denote the subcontinuum of $X_{i}$ irreducible from $x$ toa. We now show $T(x, x(a))=x$, and the Theorem then follows by Lemma 1 .

By Theorem 13 of $[1: p .272]$ either $T(x, x(a))$ has vacuous interior in $x(a)$ or it is an indecomposable continuum. In the first case, if $T(x, x(a)) \neq x$ then it contains a point $y$ and a continuum $W$ irreducible from $x$ to $y$. By hypothesis this continuum must have an essential point and hence an essential point interior to it; but $W$ has no interior point in $x(a)$ and hence none in $X$. In the second case we need only choose two points $y$ and $z$ in the same composant of $T(x, x(a))$. Then there is a subcontinuum $W$ in $T(x, x(a))$ irreducible from $y$ to $z$. By theorem 137 of $[6:$ p. 58], $W$ has no interior in $T(x, x(a))$ much less in $X$. We conclude that $T(x, x(a))=x$. By Lemma 1 , $X_{i}$ is an arc.

We denote the interior of $I$ by int $(I)$, the closure of $I$ by $\operatorname{cl}(I)$ and its boundary by $\operatorname{Fr}(I)$.

LEMMA 2. Let $X$ be a compact Hausdorff continuum and let $E$ be the set of endpoints of $X$. If (a) for each pair $a, b \in X, a \neq b$, there exists one and only one subcontinuum $I$ of $X$ irreducible from $a$ to $b$ and $\operatorname{int}(I) \neq \phi$, then (b) $X$ is hereditary both as a unicoherent and as a locally connected continuum and the $\mathrm{cl}(E)$ contains no arc.

Proof. By (1.1) of Theorem 1, $X$ is hereditarily unicoherent. Now suppose $X$ is not locally connected hereditarily, and so there exists a subcontinuum $Y$ that is not locally connected at $y \in Y$. By Wilder's Theorem 2.1 in [7: p. 102] there exist open sets $U$ and $V$ of $Y$ such that $y \in U \subset \operatorname{cl}(U) \subset V$, and there exist infinitely many components $M_{i}$ of $\operatorname{cl}(V)-U$ and a continuum $K \subset \lim \sup \left\{M_{i}\right\}$ such that $K \cdot M_{i}=\emptyset$, for all $i$, and $K \cdot \operatorname{Fr}(U) \neq \emptyset \neq$ $K \cdot \operatorname{Fr}(V)$. Now $K$ must be nondegenerate so select $a, b \in K, a \neq b$. There exists a subcontinuum $I$ of $K$ irreducible from $a$ and $b$. By the hypothesis, the interior of $I$ with respect to $X$ is non-null, hence the interior of $I$ with 
respect to $Y$ is non-null. But since $I \subset K$ and $K \subset \lim \sup \left\{M_{i}\right\}$, the interior of $I$ with respect to $Y$ is null. From this contradiction, $Y$ must be locally connected.

Suppose now the set $E$ of endpoints ${ }^{1}$ of $X$ is such that $\operatorname{cl}(E) \supset M$, an arc from $p$ to $q$. Let $p^{\prime}, q^{\prime} \in M-p-q, p^{\prime} \neq q^{\prime}$, and let $M^{\prime}$ be the subarc of $M$ from $p^{\prime}$ to $q^{\prime}$. Now $M^{\prime} \cdot E=\emptyset$ but $M^{\prime} \subset \operatorname{cl}(E)$; hence every point of $M^{\prime}$ is a limit point of $E$. Then $M^{\prime}$ has a null-interior, which contradicts the hypothesis. Hence $\mathrm{cl}(E)$ can contain no arc.

EXAmple 1. This example concerns Lemma 2 and Theorem 3. Let $a=(0,0), b=(1,0), I$ be the straight line interval from $a$ to $b$ in the cartesian plane; let $I_{i j}$ be straight line interval upward from $(i / j, 0)$ $(j=1,2, \cdots ; i=1,2, \cdots, j)$ of length $1 / j$. Let $X$ be the union of the $I_{i j}$ and $I$. Then $X$ is a dendrite with set $E$ of endpoints such that $\operatorname{cl}(E) \supset I$, the unique arc from $a$ to $b$ in $X$. However, $\operatorname{int}(I)=\emptyset$. Let now $I$, be the straight line interval from $\left(1 / 2^{j}, 0\right)$ to $\left(1 / 2^{j}, 1 / 2^{j}\right)$ and $X$ be the union of the $I$, with $I$; now $\operatorname{cl}(E)$ contains no arc.

THEOREM 3. Let $X$ be a compact Hausdorff continuum, $E$ be the set of endpoints of $X$ and $(a)$ and $(b)$ be as in Lemma 2. Then $(a)$ is a necessary and sufficient condition that (b) be true.

Proof. That (a) implies (b) was shown in Lemma 2.

Consider now the case where $X$ is hereditary both as a unicoherent and as a locally connected continuum and the $\operatorname{cl}(E)$ contains no arc. Take $a, b \in X$. By (1.1) of Theorem 1 there exists exactly one irreducible continuum. $I^{\prime}$ in $X$ from $a$ to $b$ and, since $X$ is locally connected, $I^{\prime}$ is the arc $a b$; hence $X$ is arc-wise connected. Suppose (a) is not true.

Let $p \in a b-a-b$. Since $\mathrm{cl}(E)$ does not contain an arc, we can take $p \notin \operatorname{cl}(E)$. Since $X$ is normal [7: p. 74], there exists an open set $U$ about $p$ such that $\operatorname{cl}(U) \cdot(a+b+\operatorname{cl}(E))=\emptyset$. Let $a b=a a^{\prime}+a^{\prime} p b^{\prime}+b^{\prime} b$ such that $a a^{\prime}$ and $b b^{\prime}$ are disjoint arcs and $a^{\prime} p b^{\prime}$ is the maximal subarc containing $p$ of $a b$ such that $a^{\prime} p b^{\prime}-a^{\prime}-b^{\prime} \subset U$. By normality there exists, for each $x \in a^{\prime} p b^{\prime} \cdot U$, an open set $V_{x}$ about $x$ such that $\operatorname{cl}\left(V_{x}\right) \subset U-a a^{\prime}-b b^{\prime}$. Since $\operatorname{int}(a b)=\emptyset$, there exist $y \in V_{x}-a b$ and a maximal arc $q^{\prime} y x^{\prime}$ such that $q^{\prime} \in E-a b$ and $x^{\prime} \in a b-a-b$.

Let $V_{p}^{\prime}$ be such that $\operatorname{cl}\left(V_{p}^{\prime}\right) \subset V_{p}$. Then it is necessary there exist in $V_{p}^{\prime}$ infinitely many $y_{i}$ such that the $q_{i}^{\prime} y_{i} x_{i}^{\prime}$ are mutually disjoint and $q_{i}^{\prime} \notin \operatorname{cl}\left(V_{p}\right)$. Hence there exists a limiting set $N$ of the $q_{i}^{\prime} y_{i} x_{i}^{\prime}$ such that there exist $x \in N \cdot a b \cdot \operatorname{cl}\left(V_{p}^{\prime}\right)$ and $q \in N-\operatorname{cl}\left(V_{p}^{\prime}\right)$. Therefore $X$ cannot be hereditarily locally connected, under the supposition $\operatorname{int}(a b)=\emptyset$. Hence (b) implies (a) and the Theorem is true.

1 The definition of endpoint is in $[6: p .113]$. 
Corollary 3.1. Let $X$ be a metric compact continuum, and let $E$ be the set of endpoints of $X . A$ necessary and sufficient condition that $X$ be a dendrite such that $\mathrm{cl}(E)$ contains no arc is that, for each pair of distinct points $a$ and $b$ in $X$, there exist a unique subcontinuum $I$ of $X$ irreducible from $a$ to $b$ and $\operatorname{int}(I) \neq \emptyset$.

The proof follows at once from definition $[9:$ p. 88] and from Theorem 3.

\section{Generalized hereditarily unicoherent continua}

Let $B(J)$ be a class $\left\{J_{\alpha}\right\}$ of mutually homeomorphic continua; below $J, J^{\prime}, J^{\prime \prime}$ always are elements of $B(J)$. Always $R, R^{\prime}, R^{\prime \prime}$ are regions of the compact Hausdorff space $S$. If $x \in R$, we say $R$ is about $x$; and if $X \subset M$ we say $X$ is in $M$.

We define $B(J)$-cartesian membrane, as we did to generalize an $n$-sphere in [2], as follows. Let $F$ be a compact subset of the imbedding compact Hausdorff space $S$; each $J \subset S$. Let $M$ be a subcontinuum in $F, b \in M$, and $C$ be homeomorphic to $J$. Denote by $(C \times M, b)$ the decomposition space [6: pp. 273-274] of the upper semi-continuous decomposition of the cartesion product $C \times M$, where the only nondegenerate element is taken as $C \times b$ (intuitively, the decomposition space is a sort of generalized cone with vertex at the point $C \times b)$. With this notation, we give:

Definition 1 . We say that $F$ is a $B(J)$-cartesian membrane from $b$ to $J$ if and only if there is a homeomorphism $h$ from $(C \times M, b)$ onto $F$ for some $M$ such that: (i) for some $a \in M-b, J=h(C \times a)$; (ii) for all $q \in M-b$, $h(C \times q) \in B(J)$; and (iii) $h(C \times b)=b$. If $M$ is irreducible from $a$ to $b$, then we prefix the above definition with "irreducible". We say $b$ is the vertex of $F$ and $J$ is the membrane-base, or the base, of $F$. If $M$ is a continuum, $J \subset M$ and there exists a $B(J)$-cartesian membrane $F$ in $M$ with base $J$, we say $J$ membrane-bases in $M$.

For example, let $S$ be a 2-sphere and $B(J)$ be the class of 1-spheres in $S$. Then a hemisphere $F$ is an irreducible $B(J)$-cartesian membrane with some $J \in B(J)$ as base.

Definition 1.1. A continuum $M$ is a $B(J)$-continuum if and only if: (a) there exists some $J$ in $M$; and (b) each $J$ in $M$ membrane-bases in $M$. Recall $J \in B(J)$ herein.

Definition 1.2. A $B(J)$-continum $M$ is $B(J)$-unicoherent if and only if, for every two $B(J)$-subcontinua $C$ and $C^{\prime}$ such that $M=C+C^{\prime}$ and there exists some $J$ in $C \cdot C^{\prime}$, then $C \cdot C^{\prime}$ is a $B(J)$-continuum. A $B(J)$-continuum $M$ has hereditarily a property $P$ if and only if every $B(J)$-subcontinuum in $M$ also has property $P$. 
Semigroup Example 2. Let each $C_{i}(i=1,2, \cdots, n)$ be a cone homeomorphic to the complex number disc, and with multiplication that of this disc. Let $C_{i} \cap C_{i+1}$ be the one point which is the unit in $C_{i}$, but is the zero in $C_{i+1}$, and otherwise the $C_{i}$ are disjoint; for $z \in C_{i}$ and $z^{\prime} \in C_{f}(j>i)$, let $z z^{\prime}=z^{\prime} z=z$ : this is the type of multiplication used by Hunter in Example 2, Fig. 2, of [5: p. 243]. Here $S=\cup C_{i}$ is a hereditarily $B(J)$-continuum and a topological semigroup, for $B(J)$ the class of simple closed curves in $S$ : the same is true of Hunter's Examples 1 and 2 of [5: pp. 242-243], his Example 2 has no arc at its unit element, and in neither do the elements of $B(J)$ occur everywhere densely, although they do in our $S$ above.

We wish to generalize (1.I) of Theorem 1.

We need the following Hypotheses (H1)-(H4). Let $F$ and $F^{\prime}$ be irreducible $B(J)$-cartesian membranes with $J$ as base and $b$ and $b^{\prime}$ respectively as vertices. Then:

(HI) If $F$ and $F^{\prime}$ are different membranes, then $F \neq F \cdot F^{\prime}$;

(H2) If either $F_{0}=\emptyset$ and $J^{\prime \prime}=\emptyset$, or if $F_{0}$ is a submembrane of $F$ with vertex $b$ and base $J^{\prime \prime}$ and $F_{0} \cdot F^{\prime}=\emptyset$, then each $J^{\prime}$ in $\left(F+F^{\prime}-F_{0}\right)+J^{\prime \prime}$ membrane-bases in $\left(F+F^{\prime}-F_{0}\right)+J^{\prime \prime}$;

(H3) If $J^{\prime}$ in $F \cdot F^{\prime}$ does not membrane-base in $F \cdot F^{\prime}$, then there exists a vertex $b$ of $F$ such that $b \notin F^{\prime}$; and

(H4) If $R \supset b$ and $R^{\prime} \supset b^{\prime}$, then $J$ does not membrane-base in $F+F^{\prime}-\left(R+R^{\prime}\right)$.

We are now in position to state and prove a generalization of (1.1) of Theorem 1; and (H1)-(H4) are illustrated in Examples below.

TheOREM 4. Let $S$ be a compact $B(J)$-continum and let hypotheses $(\mathrm{H1})$ and $\mathrm{H2}$ ) be satisfied. Then $S$ is hereditarily $B(J)$-unicoherent if and only if, for every $J$ in $S$, there exists a unique irreducible $B(J)$-cartesian membrane based by $J$.

Proof of necessity. Given $S$ is hereditarily $B(J)$-unicoherent; suppose $J$ in $S$ membrane-bases two different irreducible $B(J)$-cartesian membranes $F$ and $F^{\prime}$. In (H2) let $F_{0}+J^{\prime \prime}=\emptyset$; then $F+F=F$ is a $B(J)$-continuum, as are $F^{\prime}$ and $F+F^{\prime}$. In Definition 1.2 let $C=F, C^{\prime}=F^{\prime}$ and so $M=F+F^{\prime}$. Thus $J$ in $F \cdot F^{\prime}$ must membrane-base an irreducible $B(J)$-cartesian membrane $F^{\prime \prime}$ in $F \cdot F^{\prime}$ by Definition 1.2. Since $F \neq F \cdot F^{\prime}$ by (H1) and $F \cdot F^{\prime} \supset F^{\prime \prime}, F$ and $F^{\prime \prime}$ are different membranes. Hence by $(\mathrm{H} 1) F^{\prime \prime} \neq F^{\prime \prime} \cdot F$; but $F^{\prime \prime} \supset F \cdot F^{\prime \prime} \supset F^{\prime \prime}$ and so $F^{\prime \prime}=F^{\prime \prime} \cdot F$. Therefore $J$ can membranebase only one irreducible $B(J)$-cartesian membrane in $S$.

Proof of sufficiency. By hypothesis any $J$ is the base of only one $B(J)$-cartesian membrane in $S$. Suppose $S$ is not hereditarily $B(J)$-unicoherent. Then by Definition 1.2 there exist $B(J)$-continua $M, C$ and $C^{\prime}$ such 
that $S \supset M, M=C+C^{\prime}$ and there exists $J^{\prime} C C \cdot C^{\prime}$, but $C \cdot C^{\prime}$ is not a $B(J)$-continuum. Let $J C C \cdot C^{\prime}$. Since $C$ and $C^{\prime}$ are $B(J)$-continua, there exist irreducible $B(J)$-cartesian membranes, $F$ in $C$ and $F^{\prime}$ in $C^{\prime}$, and each has $J$ as base. But by hypothesis $F=F^{\prime}$, and so $C \cdot C^{\prime}$ contains $F$ with base $J$. Hence each $J$ in $C \cdot C^{\prime}$ membrane-bases in $C \cdot C^{\prime}$, and so $C \cdot C^{\prime}$ is a $B(J)$-continuum, which is a contradiction. Therefore the Theorem is true.

EXAMPLE 3.1 in which $(\mathrm{H} 1)$ and $(\mathrm{H} 4)$ do not hold. In the $(x, y)$-plane let $F_{1}$ be the closure of the interior of the triangle with vertices $(1,0),(0,0)$ and $(0,1)$ and let $F_{2}$ be defined similarly for $\left(\frac{1}{2}, 0\right),(0,0)$ and $(0,1)$; let $S=F_{1}+F_{2}$ and let $B(J)$ be the class of straight line intervals in $S$. Let $J$ be the element from $(0,0)$ to $(0,1)$. Then $F_{1}$ and $F_{2}$ can be irreducible $B(J)$-cartesian membranes with base $J$. But $F_{2}=F_{2} \cdot F_{1}$, contrary to (H1); also (H4) does not hold for this $S$. Both the necessary and the sufficient condition of Theorem 4 do not hold. Obviously, we can take $B(J)$ as a class of hereditarily indecomposable continua, and obtain a similar example.

EXAMPLE 3.2 in which the conclusion of Theorem 4 holds. In the $(x, y)$-plane let $z=(0,0), q=(1,1)$ and $z q$ be the rectilinear interval from $z$ to $q$. Let $c=(2,0)$ and from $c$ take a set $N$, homeomorphic to an arc minus one endpoint and with $z q$ as limiting set, and $N \cdot z q=\emptyset=(N-c) \cdot(x$-axis). Thus $\operatorname{cl}(N)=M$ is irreducible from $c$ to $q$. Revolve in 3-space about the $x$-axis $z q, \operatorname{cl}(N)$ and $q$, and let $F, F^{\prime}$ and $J$ be the sets so obtained. Let $S=F+F^{\prime}$ and $B(J)$ be the class of simple closed curves in $S$. Then $S$ is a hereditarily $B(J)$-unicoherent continuum. Here $F=F^{\prime} \cdot F$, contrary to the conclusion of (H1); however, $F$ is a $B(J)$-cartesian membrane with base $J$, but $F^{\prime}$ is not for $z \in M$ since (ii) of Definition 1 is not satisfied at $z$. Perhaps (HI) can be proved as a theorem when $B(J)$ is a class of $(n-1)$ spheres (we partially tried this with the stronger hypothesis of Theorem 2 in [2]).

Lemma 3. Let $F=h(C \times M, b)$ be a $B(J)$-cartesian membrane with base $J$ and let $R$ be a region about $b$. Then $R \cdot F$ contains a $B(J)$-cartesian membrane $F_{0}=h\left(C \times M^{\prime}, b\right)$ with base $h\left(C \times m_{0}\right)=J^{\prime \prime}$ such that $M^{\prime} \subset M$ and $m_{0} \in M^{\prime}$.

The proof follows without great difficulty from Definition 1: note that $C \times b$ is a point in the decomposition space $(C \times M, b)$ and $b=h(C \times b)$. Thus the proof depends upon the definition of open set in $(C \times M, b)$ about $(C \times b)$. See [6: p. 274].

Wilder in [7: p. 47] gives six properties, holding for an $n$-sphere $(n>1)$, each of which he shows is equivalent to the Phragmen-Brouwer Property, which is his Property $I^{\prime}$. We generalize Property $I^{\prime}$ in Definition 1.4, his 
Property I as Property (WI) in Definition 1.5 and his Property V as Property (WV) in Definition 1.6. This enables us to prove certain properties of a hereditarily $B(J)$-continuum, when hypotheses $(\mathrm{H} 1)-(\mathrm{H} 4)$ hold.

Definition 1.3. We say that $N$ does not $B(J)$-separate $M$, or that $M-N$ is not $B(J)$-separate, if and only if every $J$ in $M-N$ membranebases in $M-N$.

Definition 1.4. We say the $B(J)$-continuum $M$ has the $B(J)$-Phragmen-Brouwer Property if and only if, for each pair of disjoint sets $A$ and $B$ of $M$ such that $M \neq A+B$, and neither $A$ nor $B B(J)$-separates $M$, then $A+B$ does not $B(J)$-separate $M$. (We do not seem to need $A$ and $B$ closed in our proofs; in either case a 2-sphere does not have this property in contrast to Wilder's [7: pp. 47,60]).

THEOREM 5. Let $S$ be a compact $B(J)$-continuum such that $(\mathrm{H} 1)-(\mathrm{H} 4)$ hold. Then $S$ is hereditarily $B(J)$-unicoherent if and only if $S$ hereditarily has the $B(J)$-Phragmen-Brouwer Property.

Proof of the necessity (in which (H1) and (H2) are needed). Let $M$ be a $B(J)$-subcontinuum of $S$, and $A$ and $B$ be disjoint subsets such that $M \neq A+B$. Suppose $A+B$ does $B(J)$-separate $M$, while $A$ and $B$ do not. By Definition 1.3 there exists $J$ in $M-A-B$ which does not membranebase in $M-A-B$. Since $M$ is a $B(J)$-continuum, $J$ does base an irreducible $B(J)$-cartesian membrane $F$ in $M$. But $J C M-A-B$ and $M-A-B C$ $M-A$, and so by Definition $1.3 J$ membrane-bases in $M-A$, and similarly in $M-B$. Since (H1) and (H2) hold, Theorem 4 is true here. Therefore $F \subset M-A-B$, and so $J$ must membrane-base in $M-A-B$, which is a contradiction. Thus the necessity is true.

Proof of the sufficiency where (H2), (H3) and (H4) are needed. Suppose false and so there exist $B(J)$-continua $M, C$ and $C^{\prime}, M=C+C^{\prime}$, $C \cdot C^{\prime} \supset J^{\prime}$ and some $J$ in $C \cdot C^{\prime}$ does not membrane-base in $C \cdot C^{\prime}$. By Definition 1.1 there exist irreducible $B(J)$-cartesian membranes $F$ in $C$ and $F^{\prime}$ in $C^{\prime}$ and each with $J$ as base. By (H3) there exist vertices $b$ and $b^{\prime}$ of $F$ and $F^{\prime}$ respectively such that $b \notin F^{\prime}$ and $b^{\prime} \notin F$. Let $M^{\prime}=F+F^{\prime}$ and by (H2) $M^{\prime}$ is a $B(J)$-continuum. By Lemma 3 there exist irreducible submembranes $F_{0}$ and $F_{0}^{\prime}$ in $F-F \cdot F^{\prime}$ and $F^{\prime}-F \cdot F^{\prime}$ respectively. By (H2) each $J$ in $M^{\prime}-F_{0}$ membrane-bases in $M^{\prime}-F_{0}$, and similarly for $M^{\prime}-F_{0}^{\prime}$; thus by Definition 1.3 neither $F_{0}$ nor $F_{0}^{\prime} B(J)$-separates $M^{\prime}$. Hence by the $B(J)$-Phragmen-Brouwer Property $F_{0}+F_{0}^{\prime}$ does not, and so each $J$ in $M^{\prime}-F_{0}-F_{0}^{\prime}$ is a membrane-base there; this is contrary to $(\mathrm{H} 4)$ and so the Theorem is true.

Definition 1.5. We say the $B(J)$-continuum $M$ has $B(J)$-Property $(W I)$ 
if and only if, for each pair $A$ and $B$ of disjoint subsets such that $M \neq A+B$ and $J$ membrane-bases in both $M-A$ and in $M-B$, then $J$ membranebases in $M-A-B$.

Theorem 6. The $B(J)$-Property $(W I)$ is equivalent to the $B(J)$-Phragmen-Brouterer Property.

The proof follows at once from Definitions 1.3, 1.4 and 1.5.

Definition 1.6. A $B(J)$-continuum $M$ is said to have $B(J)$-Property $(W V)$ if and only if, for every $J$ in $M$ there exists subcontinuum $N$ of $M-J$ such that $J$ does not membrane-base in $M-N$.

In the Examples below let $B(J)$ be a class of 1-spheres. Then the 2sphere does not have $B(J)$-Property (WV), in contrast to Wilder's original properties [7: p. 47 and Theorem 5.19, p. 60], which however fail for the 1-sphere.

EXAmple 4.1 in which (H2) does not hold. Let $F^{\prime \prime}=h^{\prime \prime}(C \times M, b)$ be an irreducible $B(J)$-cartesian membrane with base $J=h^{\prime \prime}(C \times a)$; thus $M$ is irreducible from $a$ to $b$, and further take $M$ as follows: let $t$ and $t^{\prime}$ be arcs with only endpoints in common and such that $t+t^{\prime}=K$ is a simple closed curve in $M-$ where $M-K$ is the union of the mutually separate sets $H$ and $H^{\prime}$ and $H$ is homeomorphic to an arc minus one endpoint, as similarly is $H^{\prime}$; let $H$ have $t$ as limiting set, and similarly $H^{\prime}$ have $t^{\prime}$. Thus $F^{\prime \prime}$ contains the torus $T=h^{\prime \prime}(C \times K)$, and so $F^{\prime \prime}$ does not satisfy (H2), since for $c \in C$ $J^{\prime}=h^{\prime \prime}(c \times K)$ in $F^{\prime \prime}+F^{\prime \prime}=F^{\prime \prime}$ does not membrane-base in $F^{\prime \prime}$. Take $c_{0} \in C$ and let $E$ be a 2-cell interior to $T$ and such that $\operatorname{Fr}(E)=h^{\prime \prime}\left(c_{0} \times K\right)$. Then $S=F^{\prime \prime}+E$ is a $B(J)$-continuum, and hereditarily so. Take $c^{\prime} \in C-c_{0}$ and $c^{\prime \prime} \in C-c_{0}-c^{\prime}$. Then $h^{\prime \prime}\left(c^{\prime} \times K\right)$ is a $J^{\prime}$ which is a base for two different irreducible $B(J)$-cartesian membranes $F$ and $F^{\prime}$ such that $F+F^{\prime}=T+E$, in contradiction to the sufficiency conclusion of Theorem 4. Let $A$ be the irreducible continuum from $J$ to $T$ in $h^{\prime \prime}\left(c^{\prime} \times M\right)$, and similarly $B$ be in $h^{\prime \prime}\left(c^{\prime \prime} \times M\right)$; the $B(J)$-Phragmen-Brouwer Property does not hold for these disjoint $A$ and $B$.

EXAmPLE 4.2 in which (H2) does not hold. Recall the nature of $F^{\prime}-F$ in Example 3.2; take a set $E^{\prime}$, homeomorphic to this, in place of $E$ in Example 4.1, such that $E^{\prime}$ is interior to the torus $T$, but has $T$ as its limiting set and such that $E^{\prime}+T$ is an irreducible $B(J)$-cartesian membrane with vertex $c$ interior to the torus. Thus, for $F^{\prime \prime}$ of Example 4.1, $S=F^{\prime \prime}+E^{\prime}$ is a hereditarily $B(J)$-continuum, the conclusion of Theorem 4 holds, although (H2) does not. In both Examples 4.1 and 4.2, $S$ has hereditarily the $B(J)$ Property (WV).

Hypothesis (H.5) Let $F, F^{\prime}, F_{0} \neq \emptyset$ and $J^{\prime \prime}$ be as in (H2). Then 
$F+F^{\prime}-J^{\prime \prime}$ is the union of two mutually separate sets $F_{0}-J^{\prime \prime}$ and $F+F^{\prime}-$ $F_{0}$. $\left(F_{0}\right.$ is as in Lemma 3.)

THEOREM 7. Let $S$ be a compact $B(J)$-continuum and $(\mathrm{H} 1)-(\mathrm{H} 5)$ be true. Then $S$ is hereditarily $B(J)$-unicoherent if and only if $S$ has hereditarily the $B(J)$-Property $(W V)$.

Proof of necessity. Let $M$ be a $B(J)$-subcontinuum of $S$ and let $J C M$. Since $(\mathrm{H} 1)$ and $(\mathrm{H} 2)$ holds, Theorem 4 does, and so there exists only one irreducible $B(J)$-cartesian membrane $F$ in $M$ with base $J$. By Lemma 3 there exists submembrane $F_{0}$ in $F$ and $F_{0} \neq F$. Then $J$ does not membranebase in $M-F_{0}$, for if it did this would contradict Theorem 4. Thus in Definition 1.6 we can take $F_{0}$ as the subcontinuum $N$ of $M-J$. Therefore $S$ has hereditarily the $B(J)$-Property (WV).

Proof of the sufficiency. Let $M, C, C^{\prime}$ be $B(J)$-continua, $S \supset M$, $M=C+C^{\prime}, C \cdot C^{\prime} \supset J$, but suppose $J$ is not a membrane-base in $C \cdot C^{\prime}$. By Definition 1.1 there exist $B(J)$-cartesian membranes $F$ in $C$ and $F^{\prime}$ in $C^{\prime}$ each with base $J$; and let, by (H3), $b$ be a vertex of $F$ such that $b \notin F^{\prime}$. Let $R$ be a region about $b$ such that $F^{\prime} \cdot \operatorname{cl}(R)=\emptyset$. By Lemma 3 there exists $F_{0}$ about $b$ with base $J^{\prime \prime}$ and $F_{0} \subset R \cdot F$. By Definition 1.6 there exists a subcontinuum $N$ of $\left(F+F^{\prime}\right)-J^{\prime \prime}$ such that $J^{\prime \prime}$ does not membrane-base in $F+F^{\prime}-N$. By (H5) $J^{\prime \prime}$ separates $F+F^{\prime}$ into $F_{0}-J^{\prime \prime}$ and $F+F^{\prime}-F_{0}$, and so $N$ is contained in one of these sets. But $J^{\prime \prime}$ membrane-bases $F_{0}$ and so $N \nsubseteq F+F^{\prime}-F_{0}$; by (H2), $J^{\prime \prime}$ membrane-bases in $\left(F+F^{\prime}-F_{0}\right)+J^{\prime \prime}$ and so $N \nsubseteq F_{0}$. This is a contradiction, and so $S$ is hereditarily $B(J)$-unicoherent.

We conclude: it is difficult to describe point sets of complex construction as in [10: pp. 78-88]. In spite of losses, intuitively to do this for point set theory, there are some advantages in eliminating the algebraic machinery of [7: pp. 129-130, 209-210, etc.] by means of the topologically simpler irreducible membranes used above in generalizing "connectedness". But difficulty remains, partly hidden above by $(\mathrm{H} 1)-(\mathrm{H} 5)$, because basic definitions cannot be simple here.

The concepts above may be of interest in obtaining new characterizations (and properties) of $n$-spheres, as in part used in our paper to appear soon in Pacific Journal of Mathematics; this does illustrate some basic trouble in such an attempt.

\section{References}

[1] H. S. Davis, D. P. Stadtlander and P. M. Swingle, Semigroups, continua and the set function $T^{n}$, Duke Math. J. 29 (1962), 265-280.

[2] R. F. Dickman, L. R. Rubin and P. M. Swingle, Characterization of $n$-spheres by an excluded middle membrane principle, Michigan Math. J. 11 (1964), 53-59. 
[3] J. C. Hocking and G. S. Young, Topology (1961), Reading, Mass.

[4] R. P. Hunter, On the semigroup structure of continua, Trans. Amer. Math. Soc. 93 (1959), 356-368.

[5] R. P. Hunter, Note on semigroups, Fund. Math. 49 (1961), 233-245.

[6] R. L. Moore, Foundations of point set theory, Amer. Math. Soc. Colloquium Publication 13 (1962 revision).

[7] R. L. Wilder, Topology of manifolds, Amer. Math. Soc. Colloquium Publication 32 (1948).

[8] R. L. Wilder, Point sets in three and higher dimensions and their investigation by means of a unified analysis situs, Bull. Amer. Math. Soc. 38 (1932), 649-692.

[9] G. T. Whyburn, Analytic topology, Amer. Math. Soc. Colloquium Publication 28 (1942).

[10] P. M. Swingle, Connected sets of Wada, Michigan Math. J. 8 (1961), 77-95.

The University of Miami 